\title{
1 Global distribution and diversity of 2 prevalent sewage water plasmidomes
}

Frederik Teudt ${ }^{1}$, Saria Otani ${ }^{1}$, Frank M. Aarestrup $^{1}$

${ }^{1}$ Research Group for Genomic Epidemiology, Technical University of Denmark, Kgs. Lyngby, Denmark

Abstract

Sewage water from around the world contains an abundance of short plasmids, a number of which harbor antimicrobial resistance genes (ARGs). The global dynamics of plasmid-derived antimicrobial resistance and functions is only starting to be unveiled. Here, we utilized a previously created dataset of 159,332 assumed small plasmids from 24 different globally collected sewage samples. We investigated the detailed phylogeny as well as the interplay between their protein domains, ARGs, and predicted bacterial host genera to help understand the global sewage plasmidome dynamics.

17 A total of 58,429 circular elements carried genes encoding for plasmid-related features, and MASH distance analyses showed a very high degree of diversity. A single very diverse cluster of 520 predicted Acinetobacter plasmids was predominant among the European sewage water.

Based on functional domain network analysis, we identified three groups of plasmids, mainly replication and mobilization domains. However, these backbone domains were not exclusive to any given group. Acinetobacter was the dominant host genus among theta-replicating plasmids at these size ranges. They contained a reservoir of the macrolide resistance gene pair msr(E) and $\mathrm{mph}(\mathrm{E})$. Macrolide resistance genes were the most common resistance genes in sewage plasmidomes and found in the largest number of unique plasmids.

While $\mathrm{msr}(\mathrm{E})$ and $\mathrm{mph}(\mathrm{E})$ were limited to Acinetobacter, erm(B) was disseminated among a range of Firmicutes plasmids, including Staphylococcus and Streptococcus, highlighting a potential reservoir of antibiotics resistance for these pathogens from around the globe. 


\section{Importance}

34 Antimicrobial resistance is a global threat to human health inhibiting our ability to treat

35 infectious diseases. This study utilizes sewage water plasmidomes to identify plasmid-derived

36 features, and highlights antimicrobial resistance genes, particularly macrolide resistance to be

37 abundant in sewage water plasmidomes in Firmicutes and Acinetobacter hosts. The emergence

38 of macrolide resistance in these bacteria hints that macrolide selective pressure exists in

39 sewage water, and that the resident bacteria readily can acquire macrolide resistance via small

40 plasmids.

41

42 Keywords

43 Sewage water plasmidome, AMR, Replication initiator proteins, Bacteriophages, Metagenomics 


\section{Introduction}

46 The effectiveness of antibiotic treatment is under threat from the oncoming resistance epidemic, making antimicrobial resistance (AMR) a major problem for global health ${ }^{1}$.

The AMR epidemic is partly caused by the transmission of antimicrobial resistance genes (ARGs) between different bacterial clones and species $^{2,3}$. One of the most common and easily identifiable type of ARG-carrying mobile genetic elements is plasmids ${ }^{4}$. Plasmids often contain beneficial genes that the host microbe can use to accessorize its genome; these genes allow the microbe to adapt to a variety of factors, such as the presence of metals, antibiotics and biosynthetic and carbohydrate functions ${ }^{5,6}$.

54 Plasmids also carry genes necessary for initiating their own replication. There are three types of replication among plasmids: Rolling circle replication, theta replication, and strand displacement replication ${ }^{7}$. Rolling circle replication relies on a replication initiator to nick one of the plasmid strands creating a free strand end which is then elongated ${ }^{8}$. Plasmid theta replication and strand displacement replication is more similar to chromosomal replication: The initiator protein and/or transcription of a preprimer causes the DNA to melt at replication origins, and regular host replication forks extend RNA primers ${ }^{7}$.

Plasmids also often carry mobilization genes, allowing them to be transferred from cell to cell through mating pores. Mobilization is similar to rolling circle replication with the crucial difference that the nicked strand is not immediately replicated; instead it is transferred through a mating pore to an acceptor cell. Most mobilizable plasmids do not encode the gene necessary for mating pore formation. Instead, they rely on the mating pores provided by other plasmids. Plasmids encoding a complete mating pore are called conjugative, while those that rely on the mating pores of others are called mobilizable? .

Studies have shown an extremely high variability of plasmids within and between reservoirs ${ }^{10,11}$. An example of the former is the plasmid-encoded component of the pangenome of $E$. coli: there is far greater variation in this component than in the chromosome-encoded component of the pangenome. Additionally, the plasmid variation is not explained by pure phylogeny, but niche-phylogeny interaction ${ }^{10}$. A concrete example of how niche shapes plasmid gene content has been shown in the family Lactobacillaceae: Plasmids of bacteria adapted to living in vertebrates contain predominantly anaerobic metabolic genes, while plasmids from free-living bacteria contain aerobic metabolic genes; the availability of oxygen thus clearly affects the plasmid gene content ${ }^{11}$. It also appears that some plasmids are transmitting ARGs globally, as seen with the recent emergence of the colistin resistance gene mcr-9. This gene is found on similar IncHI2-ST1 plasmids in Europe, North America and China, suggesting a common ancestry. ${ }^{12}$ Thus, studying and understanding the global diversity and similarity of plasmids could provide novel insight into the genomic epidemiology of microbial genes, 81 including ARGs. 
Obtaining comparable samples from global sources is however, a challenge. We have recently suggested urban sewage as a comparable matrix and used that successfully to study the global occurrence of antimicrobial resistance, the virome, and human populations ${ }^{13-15}$. We recently applied long read sequencing to explore the occurrence and diversity of the small plasmidome in 24 globally obtained sewage samples and assembled 165,302 contigs (159,322 circular), of which 58,429 carried genes encoding for plasmid-related and 11,222 for virus/phage-related proteins ${ }^{16}$. These plasmidomes were compared to conventional whole community metagenomic extractions from the corresponding samples, elucidating which AMR classes was preferentially found in the plasmidome of sewage communities. One of the highlights of this article were the abundance of macrolide resistance genes in the plasmidomes, e.g. erm(B) genes, $m p h(E), \operatorname{mef}(A)$, and $m s r(D)$.

Here, we investigate these data further with the aim to find gene clusters by looking for conserved regions at domain level. We also apply machine-learning tools for linking the sequences to their most likely host and investigate the interplay between AMR, host, and backbone proteins.

\section{Material and Methods}

\section{Single read assembly}

Sewage water circular elements and their generation methods have been described in a previous publication ${ }^{16}$. Briefly, DNA was extracted from sewage samples from 24 samples from around the world (sample list in Supplemental Table 1), non-circular DNA was digested, and the circular DNA was amplified through rolling circle amplification. Libraries were prepared for both Oxford Nanopore and Illumina sequencing. Nanopore reads were trimmed, and short reads $(<10 \mathrm{~kb})$ were discarded. The raw reads consisted of tandem repeats from the rolling circle amplification step. These were assembled into the original circular sequence, which was then polished using Illumina reads with pilon ${ }^{17}$.

\section{PLSDB comparison}

108 To compare our assemblies to known plasmids, we mapped them against the plasmid database PLSDB (version 2020_11_1918) using blastn (all NCBI-blast commands were done with version NCBI-blast (this time, PLSDB as query, assemblies as subjects). If the PLSDB plasmid coverage and the assembly coverage were both above $90 \%$ for a given alignment, the pair was considered to be matching.

115 Single read assembly creates duplicates, as each read from a plasmid can give rise to a 116 sequence in the assembly. The assembly from each sample was homology reduced using kma 117 (1.3.0 $)^{20}$ : Settings were: 80\%. -k 16 -NI -Sparse - -ht 0.80 -hq 0.80 -and. Default k-mer size 16 118 was used and homology thresholds were set to $80 \%$. Homology reduction was done 
independently for each sample. The low thresholds were chosen as a countermeasure to the high error rate of Oxford Nanopore sequencing.

122 The longest 100 sequences from each of the 24 samples were individually sketched using MASH

$1232.2^{21}$ and a 2,400 by 2,400 distance table was calculated. Singletons (circular elements with

124 distance 1 to all other circular elements) were removed from the table. A neighbour-joining

125 tree was created using PHYLIP $3.697^{22}$ and visualized using Microreact ${ }^{23}$.

127 Circular elements were scanned for Pfam domains, these domain predictions have been used previously to classify elements as plasmids or phages ${ }^{16}$. Briefly, Prodigal (version 2.6.3) ${ }^{24}$ was used to predict proteins, which were scanned against the Pfam database (version 33$)^{25}$ using hmmscan from HMMER (version 3.3.1) ${ }^{26}$.

131 Pfam domains were used classify the putative proteins on the circular elements (as in our previous publication, the e-value cutoff was $0.000001^{16}$. If multiple domains with acceptable evalues were predicted in a protein, the domain with the lowest e-value was chosen. Each protein was thereby represented by its most significant domain. The number of times each domain was assessed as the most significant domain on a protein was counted. The proteins of the Comprehensive Antibiotic Resistance Database (CARD) ${ }^{27}$ protein homolog model and protein overexpression model had Pfam domains predicted in the same manner. The Pfam domains found on CARD database proteins were added to our domain classification scheme as "Potential ARG".

The combination of domains in each circular element was calculated. The amount of times a domain was found on each individual circular element was not considered. The number of times each combination was encountered in a sample was counted.

We attempted to remove phage contamination with PPR-Meta ${ }^{28}$ and SourceFinder ${ }^{29}$

144 (Supplemental Figure 1 and Supplemental Figure 2, respectively). However, neither method proved very useful. PPR-Meta falsely predicted almost half of the plasmid sequences to be

146 phage-derived. SourceFinder predicted an equally large proportion of plasmids to be either

147 chromosomal or phage-derived.

149 A bipartite network was made of the circular elements and the domains found on them. For the 150 circular elements, a node was made for each unique domain combination in each sample. For 151 example: Sixteen circular elements in the Indian sample only contained the domains Relaxase 152 and MobC; they were represented as a single node. For the domain part, each Pfam found in 153 the data was simply represented as a node. An edge was drawn between a domain and a 154 circular element if the domain was found on the circular element. To continue the example, 155 MobC and Relaxase were each represented as a node and the Indian node described above was 
connected to them both. Networks were visualized by Gephi (version 0.9 .2$)^{30}$. The size of nodes was estimated by their counts.

158 The color of the nodes were based on Pfam function. The classification scheme was adapted from previous publications ${ }^{16,31}$. The scheme was updated to include toxin-antitoxin systems as a separate group, and potential ARG domains found in the CARD databases.

The macrolide resistance gene msr(E) contains an $A B C$ transporter Pfam domain ( $A B C$ tran), thus $A B C$ _trans is marked as a potential ARG. However, not all $A B C$ _trans-containing proteins are $\mathrm{msr}(\mathrm{E})$ or ARGs. To find bona fide ARGs, the homology-reduced circular elements were aligned against the ResFinder database ${ }^{32}$ with $\mathrm{kma}^{20}$ using the following parameters: bcNano on, bc=0.7, mem_mode on.

\section{Plasmid host prediction}

168 The homology-reduced circular elements were analyzed with a random forest model plasmid host predictor created by Aytan-Aktug et al. ${ }^{33}$. The genus predicted with the highest probability was chosen as the host taxon.

\section{Length distribution of circular assemblies}

173 Sewage water plasmids from 24 samples from 22 countries around the world (Supplemental

174 Table 1), which have been described previously ${ }^{16}$, were compared to the PLSDB database. The

175 circular elements (Figure 1 top panel) were predominantly small compared to the PLSDB 176 plasmids.

177 Plasmids from PLSDB ${ }^{34}$ were generally quite large (Figure 1 bottom panel). The PLSDB plasmids

178 were split in two main types: Small plasmids of lengths of around $5 \mathrm{~kb}$, and big plasmids ranging 179 from 20 to $300 \mathrm{~kb}$. The size range of PLSDB plasmids that matched circular elements in our 180 samples were in the size range from about 1,500 bp to 5,000 bp (Figure 1 middle panel).

181 Overall, we are looking at a segment of plasmids that might be under-researched currently.

\section{Phylogenetic analyses}

183 After individual homology-reduction of the 24 samples, the longest 100 circular elements from 184 each sample were compared in phylogenetic analyses with neighbor-joining tree algorithm 185 (Figure 2). With the exception of a smaller subset of predominantly European samples indicated 186 by dotted lines, there was very little similarity between the analyzed circular elements (Figure 187 2). This was so despite the similar sizes of the tested circular elements (the largest subset from 188 each sample).

189 The 24 samples were distributed across the continents as follows: four from Africa, five from 190 Asia, eight from Europe, three from North America and four from South America. For this 
reason, overrepresentation European plasmids are expected. However, the cluster in the dotted box in Figure 2 has a higher proportion of European plasmids, than a third (which would match the proportion of samples that are from Europe). There are, however, African, Asian, North American, and South American plasmids in the cluster as well and this cluster was chosen for further downstream analyses.

Acinetobacter plasmids from sewage water

The cluster of interest chosen from Figure 2 was dominated by plasmids predicted to be Acinetobacter with only a few Neisseria or Bacillus plasmids (Figure 3A). The plasmids in the cluster were still too many for visualizing their genes and alignments between them, so an even smaller subset was selected for visualization. The subset was composed of plasmids predicted to be Acinetobacter exclusively (Figure 3A dotted circle). A majority of the plasmids was European, but all continents were still present (Figure 3B). A plasmid from each branch of the subset was annotated and aligned against the other plasmids (Figure $3 \mathrm{C}$ ).

This cluster was of interest because it contains no known replication mechanisms. This is despite the fact it contains other predominantly known plasmid-related functions, i.e., mobility systems and toxin-antitoxin systems ${ }^{35,36}$. The lack of replication proteins in these genetic elements indicates that this group of circular elements are either not plasmids, plasmids without replication initiator proteins, or plasmids with new types of replication initiator proteins.

Figure $3 C$ shows the predicted ORFs in representatives from the subset, and Table 1 lists proteins of interest. Many of the ORFs did not have a predicted Pfam associated, or they were Pfams with very uncertain function, e.g., various helix-turn-helix domains. The proteins with assignable functions involved phage protection systems (N6_N4_Mtase ${ }^{45}$, N6_Mtase ${ }^{46-48}$, AbiJ_NTD449, and Abi_C50); Cell wall metabolism (LysM ${ }^{41,42}$ and Peptidase_M15_443,44); immune escape (esiB $\left.{ }^{39}\right)$; and cell-cell signaling $\left(\mathrm{VCBS}^{40}\right)$.

\section{Clustering of circular elements into plasmids and bacteriophages}

To investigate the circular elements at large, we made a network graph of all domains to trace their interactions in the global environment. The network layout was calculated by the ForceAtlas $2^{51}$ algorithm. The layout revealed one main plasmid cluster and two clearly distinct bacteriophage clusters; the main cluster contained most of the nodes, while Phage clusters 1 and 2 were in the periphery (Figure 4). Interestingly, the two clusters were both apparently ssDNAs, as indicated by their domains ${ }^{52-54}$. One cluster contained viruses with replication domains Gemini_AL1 or Viral_Rep which may actually infect eukaryotes primarily ${ }^{53}$ (Figure 4 phage cluster 1); the other cluster contained no evident replication proteins, but did contain scaffolding protein Chlamy_scaf ${ }^{52}$ (Figure 4 phage cluster 2) and capsid domain Phage_F54

226 (pink), both found in ssDNA bacteriophages.

227 There is one last bacteriophage cluster, but it is less clearly separated from the main cluster 228 (Figure 4 phage cluster 3). The majority of the viral accessory proteins of this cluster are either 
associated with the Phage_CRI or, to a lesser extent, the Phage_X viral replication domains found in this cluster. However, some are associated with the replication initiation domains of the main plasmid cluster, explaining why phage cluster 3 fails to separate from the main cluster. This is most noticeably from the fact that the Rep_trans domain has been pulled halfway from the main cluster towards phage cluster 3 .

Plasmid replication initiators were predictably the most common domains in the dataset (Figure 4, green nodes) with mobilization domains as close second (Figure 4, blue nodes). Replication and mobilization genes separate into three clusters in the network with 2-4 major domains in each. Group 1 is composed of Rep_1, Rep_2, RepL, and Mob_Pre and is the most prevalent. Group 2 is composed of Rep_3 and MobA_MobL. Group 3 is composed of Replicase, Relaxase, and $\mathrm{MobC}$ and is the least prevalent. There were more backbone proteins, but these were selected due to two factors. First, they needed to be large enough to be identifiable in the network as a major backbone domain. Second, they needed to be unambiguously clustered with other backbone in their vicinity. Rep_trans is closest to Group 1, but it is not included in the group. Of the 72,225 circular elements, 34,659 contained Pfam domains, and 21,639 had plasmid backbone proteins from at least one of the three replication groups.

Replication group 1 contained domains for rolling circle initiator proteins (Rep_155, Rep_2 ${ }^{56,57}$, RepL ${ }^{58,59}$, and the exluded Rep_trans $\left.{ }^{60}\right)$, while groups 2 and 3 contain domains of theta replication initiators (Rep_ $3^{61,62}$ and Replicase ${ }^{63}$ ). It therefore makes sense that group 1 replication domains are the ones that are the most entangled with bacteriophages, which also use rolling circle replication ${ }^{8}$. The rolling circle-replicating section of the network generally consisted of a few large nodes, while the theta-replicating section was more dispersed (Figure 5). Most of the major backbone domains had a large cluster of circular elements connected too them: the elements with only that single backbone domain found on them. These were the most common types of circular elements.

Closer inspection of the backbone genes showed that there are minor overlap between the backbone clusters (Table 2). As expected, not all replication proteins were associated with a mobilization protein. However, if replication proteins were associated with mobilization proteins, they would predominantly belong to specific Pfams. Two different replication proteins were almost never found on the same plasmid (Table 2). Likewise, different mobilization domains were generally not found together on the same plasmid with the exception of proteins predominantly associated with Mob_Pre. 
Besides replication initiator proteins and mobilization proteins, the network also showed one other type of very common domains: toxin-antitoxin systems. They were more closely associated to the theta-replicating plasmids (Figure 5, orange nodes). Toxin-antitoxin systems were not as common as replication and mobility domains, but far more common than any other type of domains.

\section{ARGs and backbones}

After grouping the backbone proteins into three categories, the ARGs associated with each type were investigated. Table 3 shows the backbone as they occur on plasmids that carried ARGs. Interestingly, many ARGs were found on Group 1 plasmids, but not those carrying Rep_1, despite it being the most prevalent replication protein. The most prevalent backbone domain on ARG-carrying plasmids was Mob_Pre (of Group 1 backbones). The next most prevalent protein was Rep_3 (of Group 2 backbones). Overall, it was clear that ARGs associated with specific backbone proteins, rather than all of a backbone group.

Bacteriophages usually do not carry ARGs. To discover if that was the case in our samples, we investigated if there were any ResFinder hits found on the same circular elements that carried bacteriophage proteins. No bacteriophage domains were found alongside ResFinder hits. the domain network with respect to predicted host genus and sample origin. The domains RrnaAD, Aminoglyc_resit and CAT were closer associated with the rolling circle plasmids, while APH MFS_1, and ABC_trans were found with the theta-replicating plasmids. Pentapeptide_4 was located on the outskirts of the plasmid clusters. Table 4 lists which commonly found ARGs these domains correspond to.

\section{AMR and bacterial hosts}

289 The host of the ARG-carrying plasmids were also predicted in the global sewage plasmidomes 290 (Supplemental Table 3). We had a total of 150 ARGs found after homology-reduction. Using a 291 plasmid host species predictor ${ }^{33}$, we investigated the host range of ARG-carrying plasmids. The 292 ARG-carrying plasmids were limited to 14 different predicted genera.

293 Interestingly, the most common ARGs in the homology-reduced plasmids were capable of 294 conferring macrolide resistance, but in different host ranges (Supplemental Table 3). The most commonly found $A R G$ was erm(B). erm(B) is a methyltransferase that confers macrolide resistance by methylating rRNA $^{64}$. It was found in five different genera of Firmicutes. The next most common genes were the gene pair $\operatorname{msr}(E)$ and $m p h(E) . m s r(E)$ is a ribosome protection protein, conferring resistance to a wide range of ribosome-targeting antibiotics, e.g. macrolides, lincosamides, and phenicols ${ }^{65} . \mathrm{mph}(\mathrm{E})$ is a macrolide phosphotransferase, conferring resistance to macrolides ${ }^{66}$. The other AMR gene found on more than two plasmids were qnrB19 in Klebsiella and Salmonella (which confers resistance to quinolones) ${ }^{67}$; erm(T) in the Staphylococcus, Lactiplantibacillus, and Streptococcus; Inu(A) in Staphylococcus, and 
Lactiplantibacillus; tet(39) in Acinetobacter; and lastly, cat and aph(3')-III were both found in Escherichia.

Genera and backbones

Having found a link between ARG and host bacterial genera, we then investigated the relationship between the bacterial host genus and plasmid backbone type. The host predictions are listed in Supplemental Table 2. The five most often predicted bacterial host genera were analyzed for their backbone gene content. Importantly, hosts were predicted for all circular elements, including for bacteriophages. Two different methods for excluding phages were tried, but they were both too inaccurate (Supplemental Figure 1 and Supplemental Figure 2). However, bacteriophages should not have plasmid backbone genes so their output will not skew the backbone composition analysis.

Planococcus was by far the most often predicted host $(n=26,165)$, but relatively few backbone genes were found on these plasmids (Supplemental Table 4). Rep_2 and Rep_L made up an unusually large proportion of backbone genes of these plasmids. As Planococcus is predominantly a marine halophilic genus ${ }^{68,69}$ and relatively few of these circular elements carried plasmid backbone genes, it is likely that most of these predictions were false, likely stemming from phage contamination.

Neisseria was also predicted in high abundance $(n=11710$, Supplemental Table 5). The abundances of the backbone genes were fairly similar to the abundances in general, though groups 2 and 3 were again slightly underrepresented. Neisseria is a common human and animal commensal ${ }^{70}$, thus it not unexpected to find it in sewage.

Lactiplantibacillus plasmids were very similar to Neisseria plasmids, but notably contained many backbone gene combinations across groupings (e.g., Replicase and Mob_Pre, Supplemental Table 6). Lactiplantibacillus is found as a commensal ${ }^{71}$ and is used for silage production $^{72}$, thus is very likely to be found in sewage water.

Acinetobacter plasmids were had the rest a high abundance of groups 2 and 3 backbones; in fact a majority of group 3 backbones were predicted to be from Acinetobacter (Compare table Table 2 and Supplemental Table 7). Acinetobacter is fairly ubiquitous and expected in sewage 331 water ${ }^{73}$.

332 Escherichia plasmids had much fewer Rep_1 genes than the population in general, but they were otherwise fairly representative of the population (Supplemental Table 8). Escherichia is a common gut bacterium ${ }^{74}$, and thus likely present in sewage, lending credence to its prediction as a host. E. coli had by far the most varied range of backbone domains.

There was a clear link between genus and plasmid backbone genes but no genus was predicted exclusively to one group. Coloring the domain network according to predicted host genus also highlighted the association between backbone genes and host genus. Importantly, the thetareplicating plasmids were predominantly Acinetobacter plasmids while the rolling circle 
replicating plasmids were more varied in their host predictions (Supplemental Figure 3). As anticipated, the sequences predicted to be from Planococcus were not plasmids, but mainly viruses (Supplemental Figure 3, purple nodes).

\section{Geographical distribution of domains and ARGs}

344 Coloring the domain network according to continent of origin showed that the theta-replicating plasmid cluster was dominated by European samples, while sequences in phage clusters 1 and 2 were predominantly from Asian samples (Supplemental Figure 4). The rolling circle plasmids were quite varied in regarding their continent of origin.

348 The European samples contained a disproportionate amount of the most common ARGs found in the data. erm(B) and qnr19 were the only genes detected on all continents. The gene pair $\mathrm{msr}(\mathrm{E})$ and $\mathrm{mph}(\mathrm{E})$ were found on many plasmids in Europe, only a few in African samples and no other continents.

The three resistance gene most often encountered in the homology-reduced dataset all conferred macrolide resistance. erm(B) was found in Firmicutes and $\mathrm{mph}(\mathrm{E})$ and $\mathrm{msr}(\mathrm{E})$ were found in Acinetobacter. Additionally, erm(B) was found across five different genera (Supplemental Table 3). erm(B) were found on all continents. European samples contained most erm(B) genes, followed by the Americas, and the least in Africa and Asia; Africa on the other hand contained the most erm(T) genes. $\mathrm{mph}(\mathrm{E})$ and $\mathrm{msr}(\mathrm{E})$ was predominantly found in

358 Europe (Table 4).

359 The domains pentapeptide_4 (containing the gene qnrB19) and CAT (containing the gene cat)

360 were not located within replication groups. qnr19 was found in Salmonella plasmids

361 (Supplemental table 3); Salmonella plasmids was very rarely predicted in the data, but almost

362 all qnr19 genes were located on them. qnr had a worldwide distribution (Table 4). cat was

363 found on European samples (Table 4) in Escherichichia (Supplemental table 3). Plasmids

364 predicted to be Escherichia is not rare in the data, but are not associated with either 365 replication. Like CAT, Escherichia lies in between the groups in the domain network 366 (Supplemental Figure 4).

\section{Discussion}

369 In this study, we further analyzed, previously extracted and assembled short circular elements, 370 mainly plasmids, from sewage water from around the world ${ }^{16} .48 \%$ of the circular elements 371 encoded functional domains (Pfam); this low proportion was likely due to the low

372 plasmid/chromosome DNA ratio and potential low copy numbers can make it difficult to detect 373 plasmids or low quality of Oxford Nanopore reads which the plasmids were assembled from . Of

374 the Pfam-containing elements, $62 \%$ contained plasmid replication initiator proteins or 375 mobilization systems, suggesting that our findings are not artefacts. 
The lack of longer sequences above 3,000 bp (Figure 1 top panel) could be caused by a disadvantage of assembling each plasmid from a single read; in this study reads numbers tend to drop with read length, so assembly is naturally limited at higher ranges as single reads are less likely to completely span longer plasmids. The smaller peaks in the distribution could be caused by the existence of a few high-abundance plasmids or phages. There were also minor peaks in the distribution of PLSDB plasmids found in our samples (Figure 1 middle), due to redundancy in PLSDB.

The fact we see several small elements compared to PLSDB is likely due to the presence of other non-plasmid circular elements, notably bacteriophages. It could also be due to a database bias caused by lack of research into plasmids that are too small to carry genes of interest (e.g. AMR genes). However, there was no way of telling if all these elements were artefacts or true plasmids or phages, though it is unlikely that the sequences we detected functional domains on were artefacts.

\section{Sewage Acinetobacter plasmids}

Acinetobacter was a very important sewage water plasmid host in our findings; the only major cluster found in our phylogenetic analysis was composed of Acinetobacter plasmids (Figure 3A) and it was the dominant genus among Replication Groups 2 and 3 in the functional analyses(Supplemental Table 7 and Supplemental Figure 3). Acinetobacter plasmids also contained all the $\mathrm{msr}(\mathrm{E})$ and $\mathrm{mph}(\mathrm{E})$ macrolide resistance genes we found.

Of the subset of Acinetobacter plasmids that were chosen for closer analysis, a majority of them were from European samples (Figure $3 \mathrm{~B}$ ). With regards to functional domains, replication domains were the most dominant in all plasmids, however they were absent in this plasmid subset (Figure $3 \mathrm{C}$ ). It could be that the assemblies are integrative conjugative elements (ICEs). These element can exist as circular ones and contain genes for conjugation similar to plasmids, but they do not contain replication gene ${ }^{75}$. Instead, they rely on integration into the host genome for replication. At odds with this theory is the lack of any predicted integrases and excisionases between the sequences. It could also be a case of missed replication genes as there are regions of homology with no predicted ORFs (Figure 3C). Shifting the start point of the sequence does not reveal any ORF in these areas. It should be noted that only a small amount of these sequences are homologous, thus it is possible that the lack of a replication genes in this cluster is simply a coincidence. Lastly, theta-replicating plasmids, which Acinetobacter plasmids often are (Supplemental Table 7), can initiate replication with only host factors ${ }^{7}$, which could also explain the missing replication genes.

409 It made sense we found phage protection functions on our Acinetobacter plasmids, as plasmid410 encoded phage defense systems is a known strategy for combating phage infection ${ }^{76,77}$. We also found cell wall metabolism functions. Cell walls are common antibiotics targets, and the genes involved with cell wall metabolism are potential AMR genes. Specifically the Peptidase_M15_4 found on our subset (Figure $3 \mathrm{C}$ and Table 1 ) is similar to VanY, a vancomycin resistance 
414 gene ${ }^{43,44}$. However, VanY exists in a much larger gene cluster than what could possibly fit on

415 these plasmids. So if these genes somehow confer resistance to a cell wall-targeting antibiotic 416 type, it may not be vancomycin, and it is not a homologous mechanism to VanY.

\section{Functional domains in sewage plasmidomes}

418 While the findings from the inspected Acinetobacter subset were interesting, these plasmids 419 were only a small fraction of the investigated circular elements. The domain network allowed 420 us to investigate the plasmid population at large.

421 The analysis of the sewage plasmidomes at large was done based on functional Pfam

422 predictions. This methodology intrinsically filtered out very small possible artefacts from the

423 assemblies if they contained no Pfams. It also acted as a control for separating plasmids from 424 phages using domain classification.

425 The Pfam network analysis showed that there were three predominant plasmid replication 426 groups (Figure 5 and Table 2). In each group, there was an association between replication 427 domain and mobilization domain. This could be because replication and mobilization proteins 428 are dependent on the host machinery; thus, certain domains are favored by certain hosts, and 429 are therefore found together. The bacterial host genus predictions backed up that different 430 bacterial genera favor different backbone domains (Supplemental Figure 3). It was clear that there was an overrepresentation of specific combinations of genera and backbone domains. It did not appear to correlate with closely related bacterial taxa, as Planococcus plasmids (of phylum Firmicutes, Supplemental table 4) had similar backbones to Neisseria plasmids (of phylum Proteobacteria, Supplemental table 5). Backbone protein families thus do not seem to follow host phylogeny. The emergence of dominant backbone proteins within a genus must therefore have been a more recent event.

437 Replication proteins, mobility proteins, and toxin antitoxin (TA) systems were the most dominant proteins, respectively. Finding replication and mobility proteins was expected as they form the backbone of plasmids, however finding several TA systems was not expected. TA systems can be thought of as selfish genetic elements, securing the stability of the plasmid carrying it through post-segregation killing ${ }^{78}$. It could be speculated that the relative abundance of TA systems correlates to the small size distribution of our plasmids. The small size of the plasmids limits them to have barebones gene compositions. Replication genes, mobility genes and TA systems allow the plasmid to be inherited, to spread, and to secure their persistence in the host, respectively. Together these genes can be viewed as the minimal set of genes a plasmid needs to secure its survival. The small size of the plasmids studied entails that this minimal set of genes dominates the gene network (figure 5); if larger plasmids were under study, these would presumably contain a bigger proportion of genes outside of these three protein types. The role of TA systems as a third type of backbone proteins could explain why TA domains sometimes clustered together with replication groups. 
452 The clustering of ARGs in the domain network correlated with their predicted hosts

453 (Supplemental Figure 3) and their geographical origin (Supplemental Figure 4). RrnaAD and

454 Aminoglyc_resit were found in Firmicutes members, and lie in the mixed geographical cluster 455 composed of rolling circle-replicating plasmids. ABC_tran, APH, and MFS_1 were found in

456 Acinetobacter plasmids and lie in the European cluster of theta-replicating plasmids

457 (Supplemental Figure 3 and 4). The association between ARGs and genera makes sense, as not 458 all ARGs are beneficial in all bacterial genera.

459 Pentapeptide_4 (and the member gene qnrB19) was not associated with any group in the 460 domain network. Accordingly, it was associated Salmonella plasmids which were not commonly 461 predicted in the data. qnrB19-carrying plasmids seems to bear little resemblance to other 462 plasmids, but they are found world-wide, especially in the Americas. The lack of plasmids 463 similar to the qnrB19 plasmids together with the fact that Salmonella-plasmids was not 464 normally found in sewage indicate that qnrB19 was not acquired in the wastewater 465 environments. Nevertheless, their presence in the data

466 We saw no ResFinder hits on bacteriophages. This is in accordance with the finding that phage 467 and plasmid domains are well separated in the network graph with the exception of the small 468 overlap among domains for initiators of rolling circle replication.

469 The prevalence of macrolide resistance genes in sewage water across continents and species could indicate selective pressure for this trait in sewage water across the world. Though the genes $\mathrm{msr}(\mathrm{E})$ and $\mathrm{mph}(\mathrm{E})$ were almost only located in the European samples, but this could be a consequence of the composition of the microbiomes in these samples, as Acinetobacter, the carrier of the genes in sewage, was abundant in these samples. erm(B)-carrying plasmids were found worldwide, as were their host genera. It was recently indicated that methicillin resistance emerged in Staphylococcus aureus in hedgehogs as an adaptation to beta-lactam-producing dermatophyte ${ }^{79}$ highlights the coevolution among microbes in natural habitats as important sources of AMR emergence. A high percentage of antibiotics are not fully metabolized in humans, and macrolides have been detected in wastewater ${ }^{80,81}$, though wastewater treatment plants lower their abundance 81,82 .

480 It is strange that macrolide resistance selection apparently only affects Firmicutes and Acinetobacter species. It could of course be an issue of using machine learning models to predict genera; it should be kept in mind that the genera in this article are only predictions not experimentally verified. Nevertheless, it is notable that selective pressure towards macrolide resistance in sewage water seems to be a global phenomenon and it affects it affects widely different types of plasmids

487 We found that the sewage water plasmidome was functionally distinct from sewage phages. 488 There were a number combinations of plasmid backbone genes that were more common than 
others. These groups showed preferential co-occurrence with selected bacterial genera, with signals of geographical association to the sewage source, for example the higher larger amount of bacteriophages and viruses in Asian samples. Additionally, macrolide resistance-conferring plasmids were the most commonly found in the plasmid dataset. While $\mathrm{msr}(\mathrm{E})$ and $\mathrm{mph}(\mathrm{E})$ is mostly limited to Acinetobacter and European samples, erm(B) appeared on all continents, throughout a number of genera including important pathogens.

Macrolides are important antibiotics and their emergence in sewage water plasmidome could be a great threat to global health. Why macrolide resistance genes are the most prevalent is not evident, but it could be a consequence of the persistence of macrolides in wastewater. Another factor is the species composition of sewage water bacterial community. The common sewage water genus Acinetobacter carried a very large proportion of the macrolide resistance genes.

500 The prevalence of this genus in sewage water therefore to some extent explains the abundance 501 of macrolide resistance in sewage plasmidomes. Though Acinetobacter is far from the most 502 common human pathogen, the existence of a plasmid-carried reservoir of macrolide resistance 503 is a cause for concern regardless.

504 The worldwide occurrence of ARGs is an important aspect of global health management. This 505 study shows how sewage plasmidomes can be surveyed for the emergence of ARGs in the 506 environment, and points to macrolide resistance as an important emergent gene in the global 507 sewage plasmidome.

512 The authors would like to thank Derya Aytan-Aktug for help with utilizing the tools

513 PlasmidHostFinder and SourceFinder. The authors would also like to thank the two reviewers

514 for their valuable comments and suggestions. This work was mainly funded by the Novo

515 Nordisk Foundation Grant NNF160C0021856 to FMA, and partially supported by funding from

516 the European Union's Horizon 2020 Research and Innovation programme under grant

517 agreement No 773830: One Health European Joint Programme; FULL-FORCE project to FMA 518 and SO. 
522 1. World Health Organisation. Global action plan on AMR. WHO (2016).

523 2. San Millan, A. Evolution of Plasmid-Mediated Antibiotic Resistance in the Clinical

$524 \quad$ Context. Trends in Microbiology (2018). doi:10.1016/j.tim.2018.06.007

525 3. Von Wintersdorff, C. J. H. et al. Dissemination of antimicrobial resistance in microbial 526 ecosystems through horizontal gene transfer. Front. Microbiol. 7, 173 (2016).

527 4. Perry, J. A. \& Wright, G. D. The antibiotic resistance 'mobilome': Searching for the link 528 between environment and clinic. Front. Microbiol. (2013). doi:10.3389/fmicb.2013.00138

529 5. Smalla, K., Jechalke, S. \& Top, E. M. Plasmid Detection, Characterization, and Ecology. $530 \quad$ Microbiol. Spectr. 3, (2015).

531 6. Kav, A. B. et al. Insights into the bovine rumen plasmidome. Proc. Natl. Acad. Sci. 109, $532 \quad 5452-5457$ (2012).

533 7. Lilly, J. \& Camps, M. Mechanisms of Theta Plasmid Replication. Microbiol. Spectr. 3, 33$534 \quad 44$ (2015).

535 8. Wawrzyniak, P., Plucienniczak, G. \& Bartosik, D. The different faces of rolling-circle replication and its multifunctional initiator proteins. Front. Microbiol. 8, 2353 (2017).

537 9. Ramsay, J. P. et al. An updated view of plasmid conjugation and mobilization in $538 \quad$ Staphylococcus. Mob. Genet. Elements 6, e1208317 (2016).

539 10. Shaw, L. P. et al. Niche and local geography shape the pangenome of wastewater- and livestock-associated Enterobacteriaceae. Sci. Adv. 7, (2021).

11. Davray, D., Deo, D. \& Kulkarni, R. Plasmids encode niche-specific traits in Lactobacillaceae. Microb. genomics 7, (2021).

12. Li, Y. et al. Characterization of the global distribution and diversified plasmid reservoirs of the colistin resistance gene mcr-9. Sci. Reports 2020101 10, 1-10 (2020).

13. Nieuwenhuijse, D. F. et al. Setting a baseline for global urban virome surveillance in sewage. Sci. Reports 2020101 10, 1-13 (2020).

14. Hendriksen, R. S. et al. Global monitoring of antimicrobial resistance based on metagenomics analyses of urban sewage. Nat. Commun. 10, (2019).

15. Pipek, O. A. et al. Worldwide human mitochondrial haplogroup distribution from urban sewage. Sci. Rep. 9, (2019).

16. Kirstahler, P., Teudt, F., Otani, S., Aarestrup, F. M. \& Pamp, S. J. A Peek into the Plasmidome of Global Sewage. mSystems (2021). doi:10.1128/msystems.00283-21

17. Walker, B. J. et al. Pilon: An Integrated Tool for Comprehensive Microbial Variant Detection and Genome Assembly Improvement. PLoS One 9, e112963 (2014). 
18. PLSDB. Available at: https://ccb-microbe.cs.uni-saarland.de/plsdb/. (Accessed: 7th December 2021)

19. Camacho, C. et al. BLAST+: architecture and applications. BMC Bioinformatics 10, (2009).

20. Clausen, P. T. L. C., Aarestrup, F. M. \& Lund, O. Rapid and precise alignment of raw reads against redundant databases with KMA. BMC Bioinformatics (2018). doi:10.1186/s12859018-2336-6

21. Ondov, B. D. et al. Mash: fast genome and metagenome distance estimation using MinHash. Genome Biol. 2016171 17, 1-14 (2016).

22. Felsenstein, J. PHYLIP - Phylogeny Inference Package (Version 3.2). Cladistics 5, 164-166 (1989).

23. Argimón, S. et al. Microreact: visualizing and sharing data for genomic epidemiology and phylogeography. Microb. Genomics 2, e000093 (2016).

24. Hyatt, D. et al. Prodigal: Prokaryotic gene recognition and translation initiation site identification. BMC Bioinformatics (2010). doi:10.1186/1471-2105-11-119

25. Mistry, J. et al. Pfam: The protein families database in 2021. Nucleic Acids Res. (2021). doi:10.1093/nar/gkaa913

$57126 . \quad$ hmmer.org.

27. Alcock, B. P. et al. CARD 2020: Antibiotic resistome surveillance with the comprehensive antibiotic resistance database. Nucleic Acids Res. 48, D517-D525 (2020).

28. Fang, Z. et al. PPR-Meta: a tool for identifying phages and plasmids from metagenomic fragments using deep learning. Gigascience 8, (2019).

29. https://cge.cbs.dtu.dk/services/SourceFinder/.

30. Bastian, M., Heymann, S. \& Jacomy, M. Gephi: An Open Source Software for Exploring and Manipulating Networks. Third Int. AAAl Conf. Weblogs Soc. Media (2009). doi:10.1136/qshc.2004.010033

31. Jørgensen, T. S. et al. Plasmids, Viruses, And Other Circular Elements In Rat Gut. bioRxiv 143420 (2017). doi:10.1101/143420

32. Bortolaia, V. et al. ResFinder 4.0 for predictions of phenotypes from genotypes. J. Antimicrob. Chemother. 75, 3491-3500 (2020).

33. Aytan-Aktug, D. et al. PlasmidHostFinder: Prediction of plasmid hosts using random

34. Galata, V., Fehlmann, T., Backes, C. \& Keller, A. PLSDB: a resource of complete bacterial plasmids. Nucleic Acids Res. 47, D195-D202 (2019).

588 35. Hayes, F. Toxins-antitoxins: plasmid maintenance, programmed cell death, and cell cycle 
arrest. Science 301, 1496-1499 (2003).

36. Smillie, C., Garcillan-Barcia, M. P., Francia, M. V., Rocha, E. P. C. \& de la Cruz, F. Mobility of Plasmids. Microbiol. Mol. Biol. Rev. 74, 434-452 (2010).

37. Darzentas, N. Circoletto: visualizing sequence similarity with Circos. Bioinformatics 26, 2620-2621 (2010).

38. Krzywinski, M. et al. Circos: An information aesthetic for comparative genomics. Genome Res. 19, 1639-1645 (2009).

39. Pastorello, I. et al. EsiB, a novel pathogenic Escherichia coli secretory immunoglobulin Abinding protein impairing neutrophil activation. MBio 4, (2013).

40. Ginsberg, M. H., Loftus, J. C., D’Souza, S. \& Plow, E. F. Ligand binding to integrins: common and ligand specific recognition mechanisms. Cell Differ. Dev. 32, 203-213 (1990).

41. Bateman, A. \& Bycroft, M. The structure of a LysM domain from E. coli membrane-bound lytic murein transglycosylase D (MltD). J. Mol. Biol. 299, 1113-1119 (2000).

42. Joris, B. et al. Modular design of the Enterococcus hirae muramidase-2 and Streptococcus faecalis autolysin. FEMS Microbiol. Lett. 70, 257-264 (1992).

43. Evers, S. \& Courvalin, P. Regulation of VanB-type vancomycin resistance gene expression by the $\operatorname{VanS}(\mathrm{B})$-VanR (B) two-component regulatory system in Enterococcus faecalis V583. J. Bacteriol. 178, 1302-1309 (1996).

45. Timinskas, A., Butkus, V. \& Janulaitis, A. Sequence motifs characteristic for DNA

46. Schouler, C., Clier, F., Lerayer, A. L., Ehrlich, S. D. \& Chopin, M. C. A type IC restriction-

47. Schouler, C., Gautier, M., Ehrlich, S. D. \& Chopin, M. C. Combinational variation of restriction modification specificities in Lactococcus lactis. Mol. Microbiol. 28, 169-178 (1998).

49. Anantharaman, V., Makarova, K. S., Burroughs, A. M., Koonin, E. V. \& Aravind, L.

48. Thorpe, P. H., Ternent, D. \& Murray, N. E. The specificity of sty SKI, a type I restriction enzyme, implies a structure with rotational symmetry. Nucleic Acids Res. 25, 1694-1700 (1997).

Comprehensive analysis of the HEPN superfamily: identification of novel roles in intragenomic conflicts, defense, pathogenesis and RNA processing. Biol. Direct 8, (2013). 
50. Deng, Y.-M., Harvey, M. L., Liu, C.-Q. \& Dunn, N. W. A novel plasmid-encoded phage abortive infection system from Lactococcus lactis biovar. diacetylactis. FEMS Microbiol. Lett. 146, 149-154 (1997).

51. Jacomy, M., Venturini, T., Heymann, S. \& Bastian, M. ForceAtlas2, a continuous graph layout algorithm for handy network visualization designed for the Gephi software. PLoS One (2014). doi:10.1371/journal.pone.0098679

52. Tucker, K. P., Parsons, R., Symonds, E. M. \& Breitbart, M. Diversity and distribution of single-stranded DNA phages in the North Atlantic Ocean. ISME J. 5, 822 (2011).

53. Campos-Olivas, R., Louis, J. M., Clérot, D., Gronenborn, B. \& Gronenborn, A. M. The structure of a replication initiator unites diverse aspects of nucleic acid metabolism. Proc. Natl. Acad. Sci. U. S. A. 99, 10310-10315 (2002).

54. McKenna, R., Bowman, B. R., Ilag, L. L., Rossmann, M. G. \& Fane, B. A. Atomic structure of the degraded procapsid particle of the bacteriophage G4: Induced structural changes in the presence of calcium ions and functional implications. J. Mol. Biol. 256, 736-750 (1996).

55. Marsin, S. \& Forterre, P. A rolling circle replication initiator protein with a nucleotidyltransferase activity encoded by the plasmid pGT5 from the hyperthermophilic archaeon Pyrococcus abyssi. Mol. Microbiol. 27, 1183-1192 (1998).

56. Bates, E. E. M. \& Gilbert, H. J. Characterization of a cryptic plasmid from Lactobacillus plantarum. Gene 85, 253-258 (1989).

57. Xu, F., Pearce, L. E. \& Yu, P. L. Genetic analysis of a lactococcal plasmid replicon. MGG Mol. Gen. Genet. 227, 33-39 (1991).

58. Hefford, M. A., Kobayashi, Y., Allard, S. E., Forster, R. J. \& Teather, R. M. Sequence Analysis and Characterization of pOM1, a Small Cryptic Plasmid from Butyrivibrio fibrisolvens, and Its Use in Construction of a New Family of Cloning Vectors for Butyrivibrios †. Appl. Environ. Microbiol. 63, 1701-1711 (1997).

59. Lampson, B. C. \& Parisi, J. T. Nucleotide sequence of the constitutive macrolidelincosamide-streptogramin B resistance plasmid pNE131 from Staphylococcus epidermidis and homologies with Staphylococcus aureus plasmids pE194 and pSN2. J. Bacteriol. 167, 888-892 (1986).

60. Balson, D. F. \& Shaw, W. V. Nucleotide sequence of therep gene of staphylococcal plasmid pCW7. Plasmid 24, 74-80 (1990).

61. Abeles, A. L. P1 plasmid replication. Purification and DNA-binding activity of the replication protein RepA. J. Biol. Chem. 261, 3548-3555 (1986).

62. Spiers, A. J., Bhana, N. \& Bergquist, P. L. Regulatory interactions between RepA, an essential replication protein, and the DNA repeats of RepFIB from plasmid P307. J. Bacteriol. 175, 4016-4024 (1993). 
63. Giraldo, R. \& Díaz-Orejas, R. Similarities between the DNA replication initiators of Gramnegative bacteria plasmids (RepA) and eukaryotes (Orc4p)/archaea (Cdc6p). Proc. Natl. Acad. Sci. U. S. A. 98, 4938-4943 (2001).

64. Cheng, A. C. \& Jenney, A. W. J. Macrolide resistance in pneumococci-is it relevant? Pneumonia 201681 8, 1-3 (2016).

65. Sharkey, L. K. R. \& O'Neill, A. J. Antibiotic Resistance ABC-F Proteins: Bringing Target Protection into the Limelight. ACS Infect. Dis. 4, 239-246 (2018).

66. Pawlowski, A. C. et al. The evolution of substrate discrimination in macrolide antibiotic resistance enzymes. Nat. Commun. 9, (2018).

67. Cattoir, V., Nordmann, P., Silva-Sanchez, J., Espinal, P. \& Poirel, L. ISEcp1-mediated transposition of qnrB-like gene in Escherichia coli. Antimicrob. Agents Chemother. 52, 2929-2932 (2008).

68. Jung, J.-H. et al. Complete genome sequence of Planococcus sp. PAMC21323 isolated from Antarctica and its metabolic potential to detoxify pollutants. Stand. Genomic Sci. 13, (2018).

69. Waghmode, S., Suryavanshi, M., Sharma, D. \& Satpute, S. K. Planococcus Species - An Imminent Resource to Explore Biosurfactant and Bioactive Metabolites for Industrial Applications. Front. Bioeng. Biotechnol. 0, 996 (2020).

70. Liu, G., Tang, C. M. \& Exley, R. M. Non-pathogenic Neisseria: members of an abundant, multi-habitat, diverse genus. Microbiology 161, 1297-1312 (2015).

71. M, F., P, P. \& TR, K. Lactiplantibacillus plantarum-Nomad and Ideal Probiotic. Front. Microbiol. 12, (2021).

72. Bampidis, V. et al. Safety and efficacy of a feed additive consisting of Lactiplantibacillus plantarum (formerly Lactobacillus plantarum) IMI 507027 for all animal species (ALLTECHNOLOGY (IRELAND) LIMITED [Alltech Ireland]). EFSA J. 19, (2021).

73. McLellan, S. L. \& Roguet, A. The unexpected habitat in sewer pipes for the propagation of microbial communities and their imprint on urban waters. Curr. Opin. Biotechnol. 57, 3441 (2019).

74. O, T., D, S., B, P. \& E, D. The population genetics of commensal Escherichia coli. Nat. Rev. Microbiol. 8, 207-217 (2010).

75. Johnson, C. M. \& Grossman, A. D. Integrative and Conjugative Elements (ICEs): What They Do and How They Work. http://dx.doi.org/10.1146/annurev-genet-112414-055018 49, 577-601 (2015).

76. Picton, D. M. et al. The phage defence island of a multidrug resistant plasmid uses both BREX and type IV restriction for complementary protection from viruses. Nucleic Acids Res. 49, 11257-11273 (2021). 
77. Moineau, S., Walker, S. A., Holler, B. J., Vedamuthu, E. R. \& Vandenbergh, P. A. Expression of a Lactococcus lactis Phage Resistance Mechanism by Streptococcus thermophilus. Appl. Environ. Microbiol. 61, 2461-2466 (1995).

78. Jurènas, D., Fraikin, N., Goormaghtigh, F. \& Van Melderen, L. Biology and evolution of bacterial toxin-antitoxin systems. Nat. Rev. Microbiol. 2022 1-16 (2022). doi:10.1038/s41579-021-00661-1

79. Larsen, J. et al. Emergence of methicillin resistance predates the clinical use of antibiotics. Nat. 2022 44, 1-7 (2022).

80. Uluseker, C. et al. A Review on Occurrence and Spread of Antibiotic Resistance in Wastewaters and in Wastewater Treatment Plants: Mechanisms and Perspectives. Front. Microbiol. 12, 3003 (2021).

81. Pan, M. \& Yau, P. C. Fate of Macrolide Antibiotics with Different Wastewater Treatment Technologies. Water. Air. Soil Pollut. 232, (2021).

82. Rodriguez-Mozaz, S. et al. Occurrence of antibiotics and antibiotic resistance genes in hospital and urban wastewaters and their impact on the receiving river. Water Res. 69, 234-242 (2015). 
bioRxiv preprint doi: https://doi.org/10.1101/2022.02.25.482059; this version posted February 26, 2022. The copyright holder for this preprint (which was not certified by peer review) is the author/funder. All rights reserved. No reuse allowed without permission.

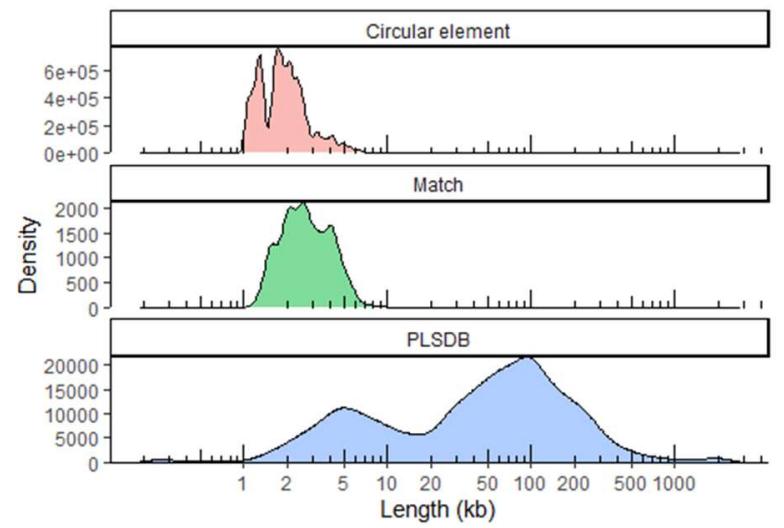

Figure 1: Length distributions of circular elements in our samples before homology reduction (top), PLSDB plasmids that match plasmids found in our samples (middle) and all PLSDB plasmids (bottom).
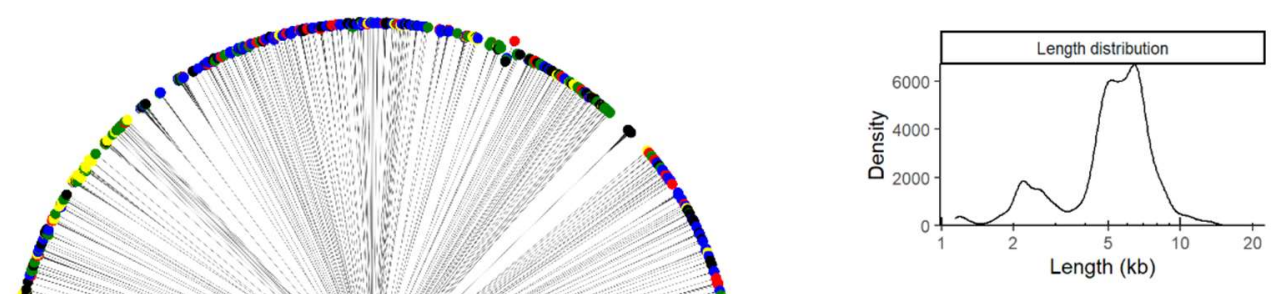

0.20

Figure 2: Dendrogram of the longest 100 circular elements from each sample. 140 singletons were removed leaving 2060 plasmids. Elements only connected through the center had no similarity. The length distribution of the circular elements is shown in the top right insert. The continent of origin of the plasmids is represented by their color. 

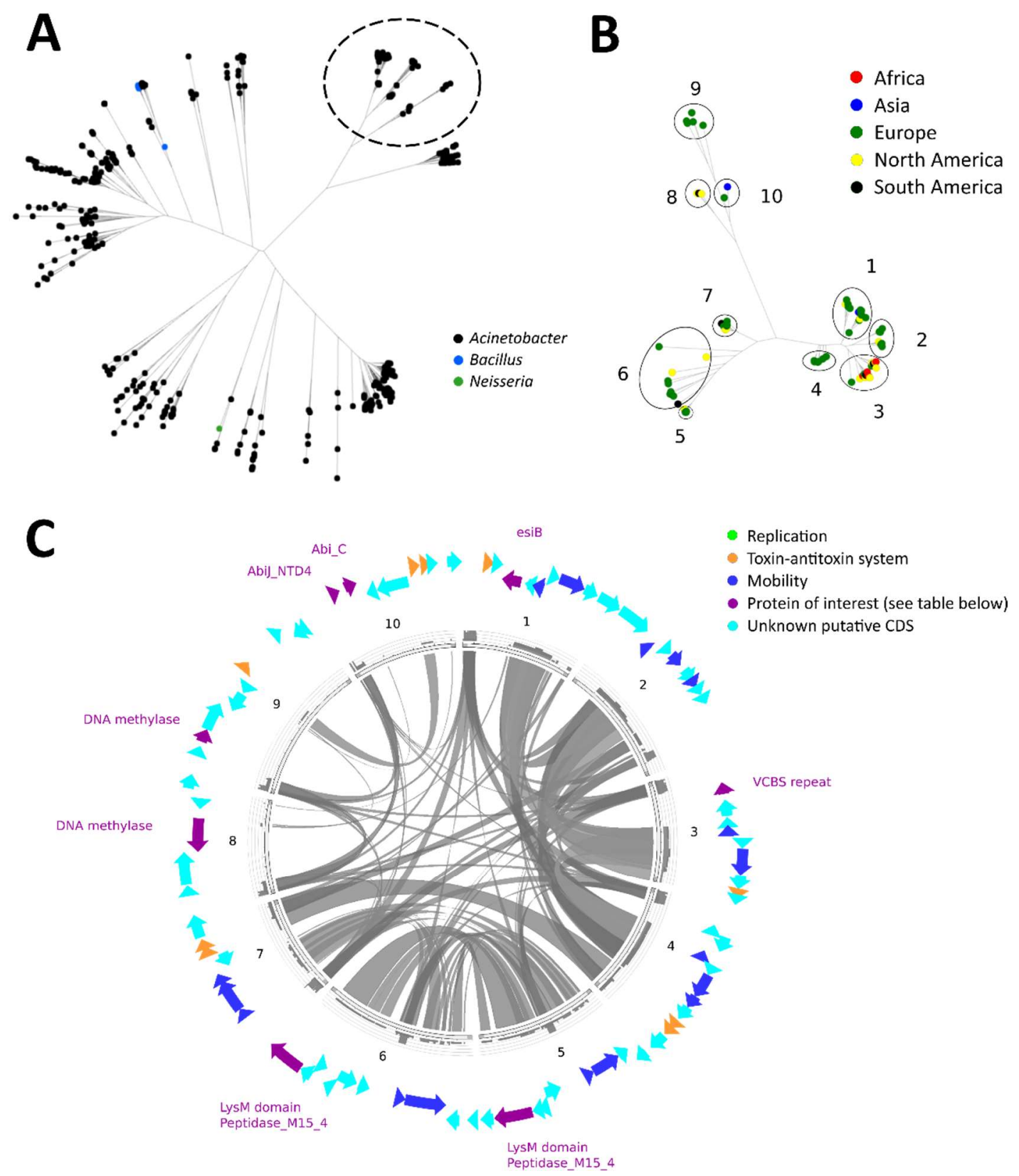

Figure 3: Visualizations of putative Acinetobacter plasmids. A: Dendrogram of the European cluster colored by genus. Subtree selected for closer inspection circled in black. B: Dendrogram showing geographical distribution of the selected Acinetobacter plasmids. Plasmids were divided into numbered groups from 1-10 for alignment-based comparison. C: Circos plots of representatives from each of the ten groups. Note that no replication genes were found. The functions of the genes highlighted in purple text is seen in Table 1. 


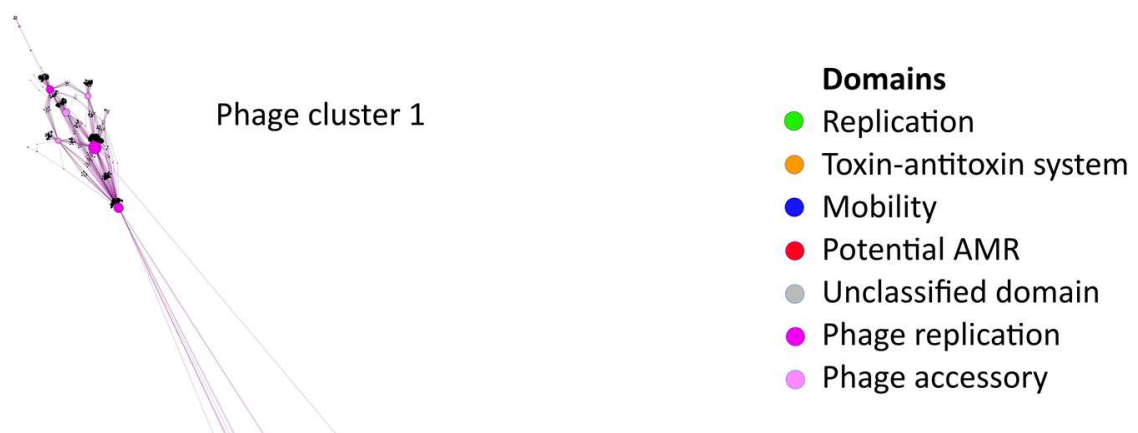

- Small circular element

Figure 4: Bipartite network graph of sewage water Pfam domains. Layout by ForceAtlas2, node sizes ordered by count. The sequences separate into four distinct clusters. A main plasmid cluster, and a viral cluster at the top (phage cluster 1 ), at the bottom (phage cluster 2), and to the left (phage cluster 3, centered around the Phage_CRI domain). Phage cluster 3 was not well separated from the plasmids due to a stronger association to the replication initiator domain of the main cluster, most noticeably Rep_trans.

\section{2}




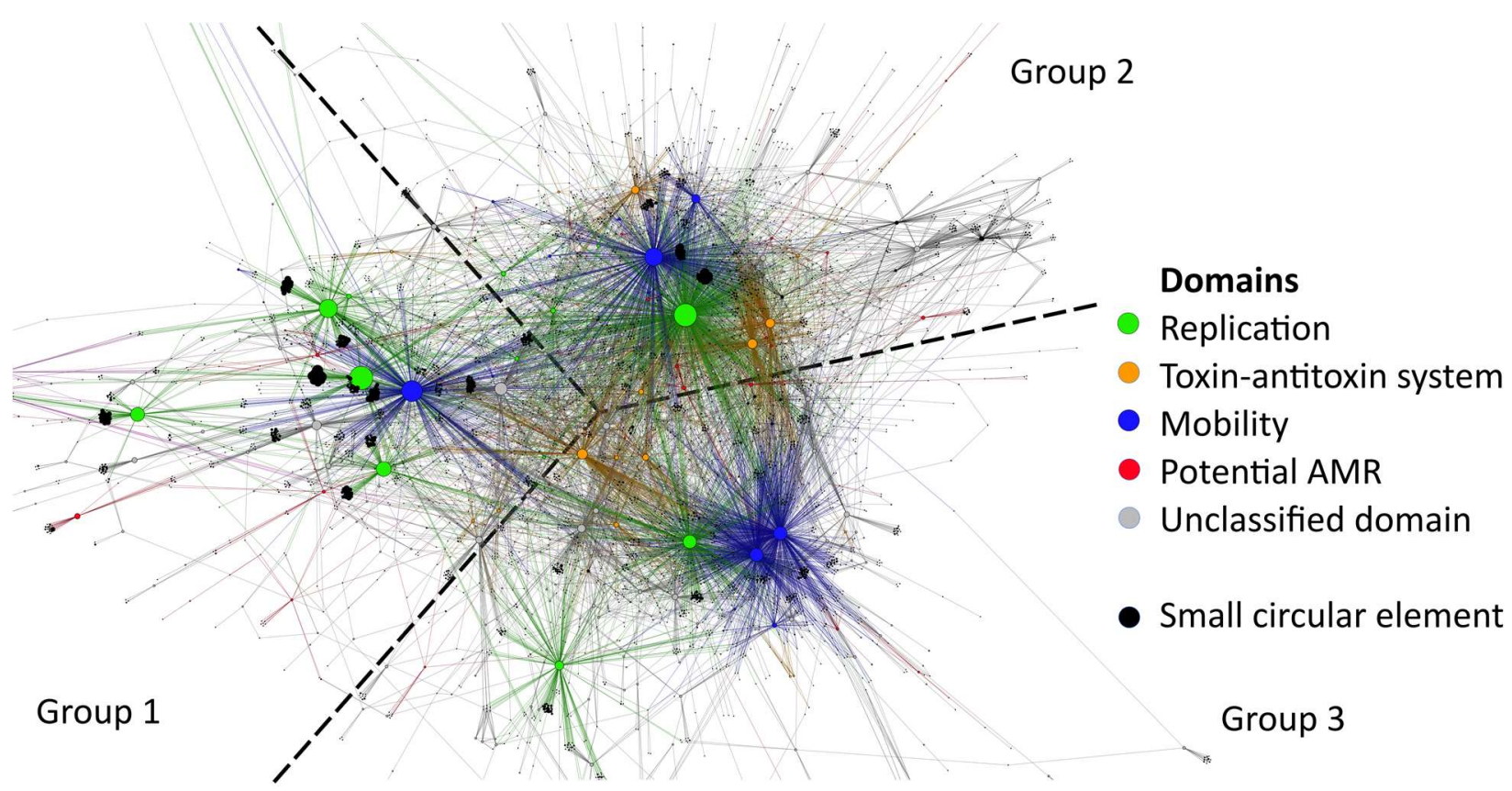

744 Figure 5: Plasmid region of bipartite network graph of sewage water Pfam domains. Layout by ForceAtlas2, node sizes by count.

745 The backbone proteins (replication and mobilization, green and blue respectively) can be separated into three groups.

748 Table 1: Protein families predicted on Acinetobacter cluster (purple CDS in Figure 3C).

\begin{tabular}{|c|c|c|}
\hline Gene or domain & Long name & Function \\
\hline EsiB & Secretory immunoglobulin A-binding protein & Inhibition of immunoglobulin A \\
\hline $\begin{array}{l}\text { PF13517 } \\
\text { VCBS } \\
\text { (FG-GAP_3) }\end{array}$ & $\begin{array}{l}\text { Repeat domain in Vibrio, Colwellia, Bradyrhizobium and } \\
\text { Shewanella } \\
\text { Also known as FG-GAP-like repeat }\end{array}$ & Diverse range of cell-cell interactions \\
\hline $\begin{array}{l}\text { PF01476 } \\
\text { LysM } \\
\text { PF13539 } \\
\text { Peptidase_M15_4 }\end{array}$ & $\begin{array}{l}\text { Lysin Motif } \\
\text { D-alanyl-D-alanine carboxypeptidase }\end{array}$ & $\begin{array}{l}\text { Peptidoglycan binding } \\
\text { Cell wall metabolism }\end{array}$ \\
\hline $\begin{array}{l}\text { PF01555 } \\
\text { N6_N4_Mtase }\end{array}$ & DNA methylase & $\begin{array}{l}\text { Host DNA protection against restriction } \\
\text { enzymes }\end{array}$ \\
\hline $\begin{array}{l}\text { PF02384 } \\
\text { N6_Mtase }\end{array}$ & N-6 DNA Methylase & $\begin{array}{l}\text { Host DNA protection against restriction } \\
\text { enzymes }\end{array}$ \\
\hline $\begin{array}{l}\text { PF18863 } \\
\text { AbiJ_NTD4 }\end{array}$ & AbiJ N-terminal domain 4 & Bacteriophage immunity \\
\hline $\begin{array}{l}\text { PF14355 } \\
\text { Abi_C }\end{array}$ & Abortive infection C-terminus & Bacteriophage immunity \\
\hline
\end{tabular}


750 Table 2: Backbone types of plasmids. Numbers indicate the amount of times two domains were observed on the same plasmid.

751 Rep_1, Rep_2, RepL, Rep_3, and Replicase are replication initiator domains. Mob_Pre, MobA_MobL, Relaxase, and MobC are

752 mobilization-related domains.

\begin{tabular}{|c|c|c|c|c|c|c|c|c|c|c|}
\hline & & \multicolumn{4}{|c|}{ Group 1} & \multicolumn{2}{|c|}{ Group 2} & \multicolumn{3}{|c|}{ Group 3} \\
\hline & & Rep_1 & Rep_2 & RepL & Mob_Pre & Rep_3 & MobA_MobL & Replicase & Relaxase & MobC \\
\hline \multirow{4}{*}{$\begin{array}{c}\text { Group } \\
1\end{array}$} & Rep_1 & 7508 & 3 & 0 & 435 & 3 & 28 & 0 & 3 & 0 \\
\hline & Rep_2 & 3 & 2508 & 1 & 895 & 11 & 0 & 0 & 5 & 2 \\
\hline & RepL & 0 & 1 & 1418 & 401 & 0 & 8 & 0 & 19 & 19 \\
\hline & Mob_Pre & 435 & 895 & 401 & 4003 & 420 & 0 & 83 & 0 & 0 \\
\hline \multirow{2}{*}{$\begin{array}{c}\text { Group } \\
2\end{array}$} & Rep_3 & 3 & 11 & 0 & 420 & 5945 & 1457 & 4 & 175 & 173 \\
\hline & MobA_MobL & 28 & 0 & 8 & 0 & 1457 & 2386 & 101 & 0 & 0 \\
\hline \multirow{3}{*}{$\begin{array}{c}\text { Group } \\
3\end{array}$} & Replicase & 0 & 0 & 0 & 83 & 4 & 101 & 1063 & 344 & 381 \\
\hline & Relaxase & 3 & 5 & 19 & 0 & 175 & 0 & 344 & 1072 & 842 \\
\hline & MobC & 0 & 2 & 19 & 0 & 173 & 0 & 381 & 842 & 1118 \\
\hline
\end{tabular}

753

754

755

756

757

758

759

760

761

762

763 Table 3 Backbone types of ARG-carrying plasmids. Numbers indicate the amount of times two domains were observed on the

764 same AMR-plasmid.

\begin{tabular}{|c|c|c|c|c|c|c|c|c|c|c|c|}
\hline & \multicolumn{4}{|c|}{ Group 1} & \multicolumn{2}{|c|}{ Group 2} & \multicolumn{4}{|c|}{ Group 3} \\
\hline & & Rep_1 & Rep_2 & RepL & Mob_Pre & Rep_3 & MobA_MobL & Replicase & Relaxase & MobC & \\
\hline \multirow{4}{*}{ Group 1} & Rep_1 & 7 & 0 & 0 & 0 & 0 & 0 & 0 & 0 & & 0 \\
\hline & Rep_2 & 0 & 27 & 0 & 21 & 2 & 0 & 0 & 0 & & 0 \\
\hline & RepL & 0 & 0 & 2 & 0 & 0 & 0 & 0 & 0 & & 0 \\
\hline & Mob_Pre & 0 & 21 & 0 & 39 & 4 & 0 & 0 & 0 & & 0 \\
\hline \multirow{2}{*}{ Group 2} & Rep_3 & 0 & 2 & 0 & 4 & 37 & 9 & 0 & 0 & & 3 \\
\hline & MobA_MobL & 0 & 0 & 0 & 0 & 9 & 9 & 0 & 0 & & 0 \\
\hline \multirow{3}{*}{ Group 3} & Replicase & 0 & 0 & 0 & 0 & 0 & 0 & 2 & 2 & & 2 \\
\hline & Relaxase & 0 & 0 & 0 & 0 & 0 & 0 & 2 & 9 & & 5 \\
\hline & MobC & 0 & 0 & 0 & 0 & 3 & 0 & 2 & 5 & & 8 \\
\hline
\end{tabular}


Table 4: Geographical distribution of the most common ARGs along with the name of their most significant Pfam domains.

\begin{tabular}{|l|c|c|c|c|c|c|c|}
\hline \multicolumn{1}{|c|}{ ARG } & Africa & Asia & Europe & $\begin{array}{l}\text { North } \\
\text { America }\end{array}$ & $\begin{array}{l}\text { South } \\
\text { America }\end{array}$ & total & Pfam \\
\hline erm(B) & 2 & 2 & 20 & 5 & 6 & 35 & RrnaAD \\
\hline $\operatorname{msr}($ E) & 3 & 0 & 17 & 0 & 0 & 20 & ABC_tran \\
\hline $\operatorname{mph}($ E) & 2 & 0 & 17 & 0 & 0 & 19 & APH \\
\hline qnrB19 & 1 & 1 & 1 & 3 & 8 & 14 & Pentapeptide_4 \\
\hline erm(T) & 4 & 0 & 3 & 1 & 0 & 8 & RrnaAD \\
\hline Inu(A) & 0 & 2 & 3 & 0 & 1 & 6 & Aminoglyc_resit \\
\hline tet(39) & 1 & 0 & 2 & 0 & 2 & 5 & MFS_1 \\
\hline aph(3')-III & 0 & 0 & 0 & 0 & 3 & 3 & APH \\
\hline $\operatorname{cat}$ & 0 & 0 & 3 & 0 & 0 & 3 & CAT \\
\hline
\end{tabular}

767 